\title{
New Pathways to Medicare Coverage for Innovative PET Radiopharmaceuticals: Report of a Medical Imaging \& Technology Alliance (MITA) Workshop
}

\author{
Bruce J. Hillman ${ }^{1}$, Richard A. Frank ${ }^{2}$, and Gail M. Rodriguez ${ }^{3}$ for the Medical Imaging \& Technology Alliance (MITA) \\ Workshop participants \\ ${ }^{1}$ University of Virginia, Charlottesville, Virginia; ${ }^{2}$ GE Healthcare, Princeton, New Jersey; and ${ }^{3}$ Medical Imaging \& Technology \\ Alliance (MITA), Rosslyn, Virginia
}

PET and PET/CT have revolutionized the diagnosis, staging, and monitoring of treatment effect or recurrence for a wide range of cancers and shown promise for improving health outcomes for patients with cardiovascular and central nervous system diseases. However, this technology is challenged by insurance coverage policies that hinder patients' access to PET and discourage technologic innovation. Recently, the Medical Imaging \& Technology Alliance (MITA), a Washington-based industry association, convened a workshop to consider new pathways for making decisions on Medicare coverage of new PET radiopharmaceuticals and imaging procedures that are currently subject to a national noncoverage decision, or "exclusionary rule." Stakeholders from the government, medical professional societies, academia, patient groups, and industry gathered to brainstorm alternatives to the national noncoverage decision and evaluate their potential to improve access and enhance innovation. Ultimately, MITA, on behalf of the PET community, expects to use the outcomes of the workshop to propose that the Centers for Medicare and Medicaid Services reconsider this current national noncoverage decision for PET and adopt a new framework for coverage.

Key Words: CMS; positron emission tomography (PET); imaging; coverage

J Nucl Med 2012; 53:336-342

DOI: 10.2967/jnumed.111.098947

$\mathbf{P}_{\mathrm{B}}$ ET (we use the term PET to include both PET and $\mathrm{PET} / \mathrm{CT}$ throughout) with ${ }^{18} \mathrm{~F}-\mathrm{FDG}$ has emerged as medicine's principal functional imaging technology. Combined with CT, PET's ability to accurately detect the physiologic changes associated with many diseases has been widely confirmed in the literature, and its use is expected to expand as evidence of its clinical utility accumulates (1). Indeed, this modality has revolutionized the diagnosis, staging, and

Received Oct. 7, 2011; revision accepted Oct. 13, 2011.

For correspondence or reprints contact: Gail M. Rodriguez, Medical Imaging \& Technology Alliance (MITA), 1300 N. 17th St., Rosslyn, VA 22209. E-mail: grodriguez@medicalimaging.org

Published online Dec. 16, 2011.

COPYRIGHT @ 2012 by the Society of Nuclear Medicine, Inc. monitoring of treatment effect or recurrence for a wide range of cancers. In addition, PET has shown promise that it will improve health outcomes for patients with cardiovascular and central nervous system diseases.

Although ${ }^{18} \mathrm{~F}-\mathrm{FDG}$ PET has improved patient care by informing changes in cancer treatment, its application is generic to the evaluation of conditions associated with altered glucose metabolism. In contrast, several PET radiopharmaceuticals in the developmental pipeline target specific molecular pathways that characterize other important physiologic processes. Examples include new agents that evaluate myocardial perfusion and molecular processes inherent in cellular proliferation, programmed cell death (apoptosis), angiogenesis, and hypoxia. Other tracers under investigation assess the pathophysiology of Alzheimer and Parkinson diseases, reflected in changes in $\beta$-amyloid and neuroreceptor density and function.

The emergence of such targeted, or mechanistic, PET tracers will fuel imaging's greater involvement in what former National Institutes of Health Director Elias Zerhouni termed P4 medicine-personalized, preventive, preemptive, and participatory health care (2). However, this compelling technologic promise is confronted by third-party insurance coverage policies that hinder patients' access to beneficial new PET radiopharmaceuticals and threaten incentives for innovation. Recently, the Medical Imaging \& Technology Alliance (MITA), a Washington-based industry association, convened a workshop to consider alternative pathways for making decisions on Medicare coverage of new PET radiopharmaceuticals and imaging procedures. Attendees included representatives of the Centers for Medicare and Medicaid Services (CMS), companies involved in developing PET technologies, patient advocates, imaging professionals (radiologists, nuclear medicine physicians, and cardiologists), health services researchers, and the relevant medical specialty societies. The purpose of the workshop was to evaluate how changes in Medicare coverage policies might better encourage innovation and improve patient access to valuable new PET technologies. Ultimately, MITA, on behalf of the PET community, expects to use the outcomes of the workshop to propose that CMS reconsider its current coverage policy for PET agents, which is unique among classes 
of pharmaceuticals: PET agents (and the PET procedures performed with these agents) that are not currently nationally covered by Medicare are subject to a national noncoverage decision, or "exclusionary rule."

\section{THE MEDICARE COVERAGE EXPERIENCE WITH 18F-FDG PET}

Medical innovations must clear multiple hurdles before their successful adoption into clinical practice. Among the most important is achieving third-party payer coverage for use of the technology in patient care. Absent such coverage, few providers will request the new procedure because patients may not be willing or able to pay out of pocket to cover its costs. In the pluralistic American health insurance environment, coverage by CMS is critically important, as private payers generally follow CMS's lead in making coverage determinations.

The usefulness of ${ }^{18} \mathrm{~F}$-FDG PET has been described for central nervous system applications since the early 1980s, for cardiac conditions since the early 1990s, and for cancer since the mid-1990s. However, PET was used infrequently in practice before 2000 largely because of limited insurance coverage.

In contrast to Medicare coverage for CT and MRI, which was left to the discretion of local Medicare contractors and generally was quite broad, CMS determined that coverage for PET would be defined for the entire nation by its national coverage determination (NCD) process. (Coverage for the great majority of innovations is dealt with by 16 regional health insurance carriers-CMS contractorswhich independently make local coverage determinations [LCDs] for the individuals serviced by CMS in that region. However, for particularly high-impact technologies, CMS may dictate an NCD. An NCD supersedes any LCD.) The CMS coverage policy for PET is an exclusionary policy, which means that if a particular indication is not explicitly listed as covered, it is not covered nationally. Moreover, CMS did not cover PET broadly but instead made its coverage decisions on an indication-by-indication basis (and, in the case of oncologic use, on a cancer-by-cancer basis). (Clinical applications include diagnosis, staging, evaluation of response during treatment, restaging [detection of residual disease] after treatment, and detection of suspected recurrence.) Because of this national noncoverage determination and iterative submission requirements for coverage by both cancer type and clinical application, it was not until 1998 that CMS approved reimbursement for the first oncologic applications: diagnostic evaluation of solitary pulmonary nodules and initial staging of non-small cell lung cancer (3). A steadily increasing number of small, singlesite studies suggested a larger role for PET in cancer care, but few generalizable, multicenter clinical trials were conducted to support broadening indications for PET coverage by CMS. In part, this phenomenon is a result of the nonproprietary nature of ${ }^{18} \mathrm{~F}-\mathrm{FDG}$; as a generic tracer manufactured and distributed by many firms, its nonexclusivity and accompanying pricing pressures limited enthusiasm for the expensive, large-scale research necessary to support traditional applications for coverage.

Between 1998 and 2004, CMS approved several additional oncologic indications for PET, with each incremental change in coverage requiring adherence to the full $\mathrm{NCD}$ process. In January 2005, CMS indicated its willingness to consider expanding coverage for oncologic indications based on the performance of ${ }^{18} \mathrm{~F}$-FDG PET in the care of patients enrolled in a clinical trial or registry. This policy, known as "coverage with evidence development" (CED) (4), would allow for payments to providers contributing PET data to inform future coverage decisions.

In response, Drs. Barry Siegel and R. Edward Coleman proposed the development of a national registry for ${ }^{18} \mathrm{~F}-\mathrm{FDG}$ PET that would assess its value in guiding clinical decision making in cancer patients. CMS agreed that demonstrating that PET led to changes in intended patient management would serve as a reasonable proxy for what the agency really wanted to know: for which, if any, cancers and clinical indications did use of ${ }^{18} \mathrm{~F}-\mathrm{FDG}$ PET lead to improved health outcomes for Medicare beneficiaries? (During the workshop, Dr. Louis Jacques, the CMS director for coverage and analysis, cited the following as CMS's preferred "road to diagnostic coverage": "Provide adequate evidence that the incremental information obtained by the new diagnostic technology, compared with alternatives, changes physician recommendations resulting in changes in therapy that improve clinically meaningful health outcomes in Medicare beneficiaries.") The registry that was developed in response to CMS's CED policy became known as the National Oncologic PET Registry (NOPR). It was anticipated that CMS would decide on coverage for cancer-indication pairs as sufficient registry data became available to support a "yes" or "no" decision (5).

Drs. Siegel and Coleman recruited Dr. Bruce Hillner to lead the NOPR research effort. Dr. Hillner had previously published a single-institution study demonstrating the feasibility of studying the effect of ${ }^{18} \mathrm{~F}-\mathrm{FDG}$ PET on altering physicians' therapeutic intent (6). A coalition of professional organizations supported the NOPR launch: the Academy of Molecular Imaging, the primary sponsor of the NOPR; the American College of Radiology Imaging Network, which was the vehicle for scientific and infrastructure development and for the day-to-day management of the registry; the American Society for Clinical Oncology; the American College of Radiology; and the Society of Nuclear Medicine. All NOPR activities, including provider registration, case submission, and credit card payment for data submission, traversed a Web portal developed and serviced by the clinical trials cooperative group American College of Radiology Imaging Network. (Each provider paid a 1-time fee of $\$ 50$ to register and $\$ 50$ for each submitted case. Upon submission of the data for a case, the provider would receive payment from CMS for the PET scan. Ultimately, NOPR paid for itself.)

The primary data collected by the NOPR were derived from questionnaires completed by referring physicians indicating their patient management intentions before and after 
receiving the results of PET scans ordered for their cancer patients. From the initiation of NOPR in May 2006 to April 2009 , nearly 1,900 PET providers submitted almost 133,000 cases to the registry. Using this large volume of data, the NOPR investigators published several peer-reviewed papers describing the major findings of the registry. In April 2009, after consideration of the NOPR data and a health technology assessment requested by CMS and reviewed by the Medicare Coverage Analysis Committee, CMS greatly expanded PET coverage to additional cancers and clinical indications, as listed in a table published on the NOPR Web site (7). NOPR's contributions to PET coverage were significant. They proved the feasibility and utility of CED in demonstrating the value of PET to cancer care and established a collaborative relationship among imaging providers and their referring physicians to supply CED data. NOPR facilitated access to PET for patients who otherwise would have been unable to afford scans, offered clinical evidence confirming the considerable body of published work suggesting that PET was reasonable and necessary, and ultimately justified broader coverage for PET scans.

\section{THE MITA PET COVERAGE WORKSHOP: ALTERNATIVES TO CURRENT CMS COVERAGE PROCESSES}

Despite the important contributions of NOPR, the time and resources required to achieve broad PET coverage and enhance Medicare beneficiaries' access to PET through CED was burdensome and expensive. Concern over a reprise of the ${ }^{18} \mathrm{~F}-\mathrm{FDG}$ experience in bringing a new PET radiopharmaceutical to market potentially discourages companies from pursuing the development of new agents that would enhance patient care for several critical diseases. Indeed, the current exclusionary policy would not permit coverage or payment for new radiopharmaceuticals outside the formal NCD process, indication by indication.

The MITA PET Coverage Workshop was convened to develop possible alternatives to the noncoverage policy for PET. Experts and stakeholders from a wide variety of backgrounds participated (Appendix).

The workshop began with a four hour brainstorming session to elicit creative ideas and alternatives to the current policy. By the end of the day, workshop participants had conceptualized 5 distinct alternatives to the noncoverage decision. Attendees were then assigned to breakout teams, which were directed to flesh out the following options into practical proposals during the final session the next day:

- Reform of the NCD process to improve responsiveness and timeliness;

- Parallel review of new radiopharmaceuticals by the Food and Drug Administration (FDA) and CMS without delay to initial marketing approval;

- Coverage by LCD instead of NCD, consistent with the method by which most other innovations are covered;
- Coverage with immediate effect from FDA approval, with relevant conditions and sponsor commitments; and

- Multiple pathways.

The following morning, workshop participants gathered for a series of presentations designed to provide background for the actual work of transforming the 5 alternatives into detailed policy proposals. The faculty's thoughtful presentations set the stage for informed analysis of the 5 options by the breakout teams that afternoon (Appendix). During this didactic session, Dr. Louis Jacques, head of the CMS Coverage and Analysis Group, shared the history and status of coverage for PET, along with the CMS Coverage and Analysis Group's expectations for "reasonable and necessary" evidence requirements. Specifically, Dr. Jacques reiterated the following conditions for CMS to consider a coverage pathway (Louis Jacques, oral communication, April 8, 2011):

1. Ensure CMS's confidence in the drug under review, the methods used, etc.

2. Define coverage for appropriate populations: set boundaries so that CMS does not pay "full freight up front."

3. Include the ability to withdraw or modify payment if expectations are not met.

The value of an alternative pathway or pathways to coverage may be rendered moot if the clinical evidentiary standards are unachievable. If, for example, a diagnostic procedure is expected to demonstrate outcomes typically associated with therapeutic interventions, such as mortality reduction, such a standard would be unreasonable given the difficulty of demonstrating the impact of an imaging agent (and the imaging test performed with that agent) through a complex chain of diagnostic and therapeutic interventions to an ultimate patient outcome such as death. The change in therapeutic planning resulting from a diagnostic test such as ${ }^{18} \mathrm{~F}-\mathrm{FDG}$ PET is sometimes a change to fewer medical interventions and perhaps to watchful waiting or palliative care. A hierarchy of clinical evidence suitable for the investigation of new diagnostic imaging agents was recently proposed by Gazelle et al. (8) and bears consideration in any discussion of a resolution to the PET noncoverage decision (see also Frank et al. 2011 (9)).

\section{CONSIDERATIONS OF THE WORKING GROUPS}

On the second day of the workshop, participants broke out into assigned teams, with a single coverage option assigned to each team. The teams were charged with considering 7 working questions to the extent that they were important to their assigned option for a new pathway to coverage:

1. What are the special features and advantages commending the option? 
2. What are the evidentiary requirements needed to support coverage?

3. How well does the option satisfy CMS's coverage criteria?

a. Build confidence that the candidate radiopharmaceutical or PET procedure is reasonable and necessary.

b. Define the population and applications for which the radiopharmaceutical or PET procedure might be appropriately applied.

c. Enable withdrawal or modification of coverage if one or more specific applications are shown to be inappropriate.

4. What are the timeline and potential obstacles or problems?

5. Are there any differences inherent in the option between proprietary and nonproprietary radiopharmaceuticals?

6 . What changes in the language of existing regulations might be necessary to implement the option?

7. Will there be any need for postcoverage surveillance, and if so, what is the mechanism to accomplish it?

Responses to these 7 questions would provide the framework for evaluating each of the options. What follows is a synopsis of each option.

\section{Group 1: NCD Reform}

Group 1 was charged with examining how a reform of the current NCD process could result in more efficient, less burdensome coverage decisions for new PET radiopharmaceuticals. In response, the participants developed a "straw man" NCD process:

- The national noncoverage decision (exclusionary rule) for new PET tracers could be reconsidered. Medicare's current NCD for PET scans states that "a particular use of PET scans is not covered unless this manual specifically provides that such use is covered." This means that any new PET tracer will remain noncovered until CMS undertakes a 6- to 12-mo evidence review of the new tracer. Unfortunately, Medicare's capacity for such new NCDs is constrained by its limited staff and the need for coverage decisions across all aspects of health care. The first step in NCD reform would thus be removal of the current blanket restriction on coverage. This would occur even for products for which data have never been reviewed-that is, FDAapproved products and indications that have never been found to be less than reasonable and necessary.

- CMS could provide national guidance on what data are necessary for the agency to decide what technologies are medically reasonable and necessary. For example, 3 broad categories could be introduced, in ascending order of scrutiny: first, differential diagnosis for initial treatment planning; second, improved accuracy over existing clinical options; and third, therapeutic monitoring for individualization of treatment regimens.

- Ordering and billing CMS for PET scans using a new tracer would represent the view of the patient care team that the test in question was reasonable and necessary for patient care under at least 1 of the above 3 rationales. Like all current imaging modalities, reimbursed scans would be subject to auditing for overutilization.

- The coverage status of a tracer and its context for use would be reviewable by Medicare at a later time and subject to change based on subsequent publication and auditing of clinical use.

- CMS could choose to forego an NCD in favor of regional LCD processes.

The main advantage of this approach is that coverage would occur quickly in accordance with the practice of medicine locally but could be modified or revoked if performance in the clinical setting was poor or subject to inappropriate use.

\section{Group 2: LCD}

Group 2 considered how Medicare coverage for most new PET radiopharmaceuticals and PET procedures could be addressed through LCDs.

- LCD is a faster, more nimble process than NCD and enables the normal practice of medicine by early adopters of FDA-approved innovations, providing access to patients in need.

- Regional carriers regularly review use to protect against inappropriate use.

- National coverage decisions could then be made on the basis of observed utility among regional carriers, taking into account important variations among regions.

- A request to supplant an NCD with an LCD could come from any interested party.

A principal concern with the LCD approach is the potential variability in coverage that may occur. However, the richness of this experience by early adopters will inform coverage decisions, as has been the case for many other products and procedures covered by CMS today. In addition, it is expected that professional societies would develop consensus guidelines that could help to achieve homogeneity among the LCDs of different Medicare administrative contractors.

\section{Group 3: FDA-CMS Parallel Review}

Group 3 assessed the potential for improvement in processes proffered by simultaneous review by FDA and CMS without extended timelines. Under a recent FDACMS memorandum of understanding introducing the concept of sharing data between the 2 agencies, CMS requested public comment on the concept of "parallel review" (10). Under parallel review, CMS would launch a national coverage analysis to determine whether the product is reasonable and necessary for the Medicare population. At the same time, FDA would pursue its premarket review to determine whether the product meets applicable safety and effectiveness standards. The process would feature the following conditions: 
- Parallel review would be voluntary and proceed only at the request of the sponsor. FDA and CMS would share information only under conditions that would protect sponsors' confidentiality.

- The nature of the evidence required for a coverage determination would be agreed to in advance.

- A flexible strategy would be necessary to facilitate adaptability as new information appears. If evidence is better or worse than expected, coverage could be expanded or contracted.

- The evidence to support FDA and CMS reviews could be obtained via the same or different scientific evidence. The type and amount of evidence required for CMS approval would depend on the role of the agent or test (e.g., for detection, diagnosis, prognosis, prediction, or monitoring), the prevalence of the disease, and whether the new test adds to or replaces existing tests.

- Modeling could be useful in extrapolating or generalizing available data.

- Assuming the evidence satisfied the predetermined criteria, CMS coverage would commence for a predetermined period after FDA approval and end after a specified period unless the postmarketing requirements were met.

- Provisional coverage would be reevaluated by CMS after real-world experience with clinical use. Coverage could be modified on the basis of the results of postmarketing evaluation, the terms of which would be agreed to by CMS and the sponsor and could include publication requirements (i.e., data published within a predetermined period), formal review of data with CMS, a registry for FDA label indications, other off-label uses, modeling, or phase 4 studies.

The parallel review approach would allow for earlier coverage decisions and might reduce the resources required of the sponsor. However, failure to obtain FDA approval to market the agent would mean that company and CMS expenditures specifically directed toward CMS evaluation would be wasted. In addition, alignment of the FDA and CMS evidentiary goals may prove difficult.

\section{Group 4: Coverage with Immediate Effect Subject to Continuing Review and Revised Determination}

Group 4 proposed conditions under which provisional coverage for a new PET tracer would be granted on the sponsor's application. This team developed a methodology that could lead to more rapid or immediate (post-FDA approval) coverage of new PET agents or indications by addressing CMS's key needs. The process involves a longitudinal relationship with CMS, starting with agreement on the nature of the data needed to approve coverage, continuing from application through provisional coverage, and finally including postcoverage auditing. Coverage with immediate effect would be conditioned on the following:
- CMS's consideration and evaluation of coverage would occur coincident with FDA review but would not be expected or implemented until FDA approval.

- The NCD would be opened only once. Subsequent future indications could be considered without requiring another NCD.

- CMS would grant provisional coverage (e.g., via temporary codes, which are widely used for other modalities) on the proviso that data that are more relevant to CMS (e.g., outcomes, impact on therapeutic decision making, and Medicare demographics) would be gathered after approval to support continued coverage.

- The sponsor and CMS would meet well before product approval and collaborate to achieve an agreement on the type of data needed to ensure continued coverage. This process might resemble FDA's "Special Protocol Assessment" process, which is currently used by sponsors to gain agreement with that agency on the registration study, endpoints, and types of data needed to support approval.

- Sponsors would be responsible for generating the agreed-on evidence after coverage, within a committed time frame.

- Based on characteristics of the submission, a "staged evidence-generation" approach might be appropriate. The following evidence hierarchy could be implemented, in part or in full, depending on the level of evidence needed to demonstrate meaningful impact: hypothesis-driven models; decision simulations using phase III data; registries; expanded registries or assessment of patient outcomes (perhaps using Medicare claims data); randomized controlled trials.

- A postmarketing use audit may be conducted to assure CMS that the technology was being used appropriately. Reevaluation and revision of coverage determination would be based on use data.

The principal virtue of this approach would be greatly accelerated patient access to new PET radiopharmaceuticals. It would provide an incentive for the sponsor to quickly accrue appropriate data to support continuing coverage. The close monitoring and iterative planning would build strong industry-CMS partnership in developing the clinical evidence necessary to demonstrate reasonableness and necessity.

\section{Group 5: Multiple Coverage Determination Pathways}

Group 5 evaluated a coverage determination model that would be variable in speed and rigor based on characteristics of both the novel tracer and the clinical application for which the product is intended.

- Radiopharmaceuticals for which early clinical use suggests the possibility of a significant impact on improving care or outcomes for high-prevalence and highmorbidity or -mortality conditions would be hurried into 
clinical care, perhaps via LCD. Such situations would require that sponsors generate the most rigorous evidence during a provisional coverage and evaluation period.

- New tracers that represent modest improvements on extant diagnostic methods or ones that were applicable to less serious conditions would undergo greater scrutiny from the outset (perhaps via NCD with or without CED) to ensure they would not simply promote additive use with little patient benefit.

- The types of data required to sustain coverage would be chosen to fit the circumstances and the extent of previous clinical research. The greatest evidentiary onus would be on applications for products having the farthest-reaching potential for improving health outcomes. Possible data sources, in descending order of rigor, might include randomized controlled trials, nonrandomized trials, registries, modeling, metaanalysis of the existing literature, and unpublished clinical experience.

This approach would offer CMS maximum flexibility in dealing with PET radiopharmaceuticals having a broad range of utility, hastening the potentially most valuable innovations to the bedside. However, 2 major concerns emerge with this approach: who would determine the assignment to a pathway, and what recourse would the sponsor have in challenging the assignment if the sponsor deemed it inappropriate?

\section{CONCLUSION}

All MITA PET Coverage Workshop participants concluded that each of the 5 proposed coverage options was worthy of further consideration. Each alternative features advantages and disadvantages, and it is expected that further analysis will culminate in a consensus that 1 or 2 preferred options would yield the best results for the PET community, including CMS and its beneficiaries.

Ultimately, this analysis will be used to make a formal MITA request that CMS reconsider the current exclusionary policy of NCD for PET and to recommend consensus pathways to CMS coverage for new PET radiopharmaceuticals and procedures. Working together with CMS to derive and implement such a solution would better encourage the development of innovative radiotracers and improve Medicare beneficiary access to clinically important and valuable PET scans.

\section{APPENDIX}

\section{Participating Agencies and Organizations}

Academy of Molecular Imaging

Agency for Healthcare Research and Quality

Alzheimer's Association

American College of Radiology

American College of Radiology Imaging Network
American Society of Nuclear Cardiology

Center for Medical Technology Policy

CMS

Council on Radionuclides and Radiopharmaceuticals MITA

Representatives from MITA member companies

National Comprehensive Cancer Network

NOPR

Ovarian Cancer Alliance

Radiological Society of North America

Society of Nuclear Medicine

\section{Workshop Participants}

Brian Abraham, IBA Group; Reza Alavi, Center for Medical Technology Policy; Timothy Bateman, American Society of Nuclear Cardiology; Gary Bertetti, Bayer Healthcare Pharmaceuticals; Brian Bresnahan, University of Washington; Julian Brunner, Center for Medical Technology Policy; Sue Bunning, Society of Nuclear Medicine; Stuart Caplan, CMS; Brian Carey, Foley Hoag, Academy of Molecular Imaging; Lawrence Clark, Highmark; R. Edward Coleman, NOPR; Dominique Delbeke, National Comprehensive Cancer Network Specialized Imaging Research Consortium, Society of Nuclear Medicine; Gary Dillehay, Society of Nuclear Medicine; Kathy Flood, American Society of Nuclear Cardiology; Richard Frank, GE Healthcare, MITA; Constantine Gatsonis, Brown University; James Gilroy, Eli Lilly and Company; Colleen Glynn, Cardinal Health; Rosemarie Hakim, CMS; Gary Heller, American Society of Nuclear Cardiology; Bruce Hillman, American College of Radiology; Bruce Hillner, NOPR; Craig Hunter, Eli Lilly and Company; Louis Jacques, CMS; Norman LaFrance, IBA Group; Jon Larson, National Comprehensive Cancer Network; William Lawrence, Agency for Healthcare Research and Quality; Jane Majcher, GE Healthcare, Council on Radionuclides and Radiopharmaceuticals; Bill McGivney, National Comprehensive Cancer Network; Anita McGlothlin, American College of Radiology; Denise Merlino, Merlino Healthcare Consulting; Virginia Pappas, Society of Nuclear Medicine; Adrienne Pavan, Bayer Healthcare Pharmaceuticals; David Pendleton, Lantheus Medical Imaging; Laurel Pracht, Ovarian Cancer Alliance; Bruce Quinn, Foley Hoag, Academy of Molecular Imaging; Mary Richards, Alzheimer's Association; Jeffrey Roche, CMS; Gail M. Rodriguez, MITA; Don Rucker, Siemens Medical Solutions USA; Lisa Saake, Covidien, Council on Radionuclides and Radiopharmaceuticals; George Segall, Society of Nuclear Medicine; Wei Li Shao, Eli Lilly and Company; Leslee Shaw, American Society of Nuclear Cardiology; Barry Siegel, American College of Radiology Imaging Network; Dan Sullivan, Radiological Society of North America; Thomas H. Tulip, Neoprobe, MITA; Rich Wahl, Johns Hopkins; Peter Webner, Positron; Terri Wilson, Siemens, PETNET Solutions, MITA; Anne Marie Wouters, Manatt Health Solutions; Anthony Yudd, American College of Radiology; Robert Zeman, American College of Radiology 


\section{Faculty and Presentation Topics}

George Segall, VA Palo Alto Health Care System, Stanford University, Society of Nuclear Medicine: "PET Imaging Horizon Scanning."

Louis Jacques, Coverage and Analysis Group at CMS:

"Evidence for Decision-Making: A PET Perspective."

William Lawrence, Center for Outcomes and Evidence at the Agency for Healthcare Research and Quality: "Evidence in Imaging."

Bruce E. Hillner, Virginia Commonwealth University: "Expectations for Outcomes and Endpoints in Diagnostics: Lessons from NOPR."

Constantine Gatsonis, Center for Statistical Sciences at Brown University: "Evidence Generalizability and Inference."

Brian Bresnahan, University of Washington: "Economic Considerations for Diagnostic Imaging."

\section{ACKNOWLEDGMENTS}

We thank the following individuals for their valuable contributions to this article: R. Edward Coleman, Jane Majcher, David B. Pendleton, Bruce Quinn, Barry A. Siegel, Daniel Sullivan, and Terri B. Wilson.

\section{REFERENCES}

1. Gambhir SS, Czernin J, Schwimmer J, et al. A tabulated summary of the FDG PET literature. J Nucl Med. 2001;42(suppl):1S-93S.

2. Zerhouni EA. Major trends in the imaging sciences: 2007 Eugene P. Pendergrass New Horizons Lecture. Radiology. 2008;249:403-409.

3. National Coverage Determination (NCD) for PET Scans (220.6). Centers for Medicare and Medicaid Services Web site. Available at: https:/www.cms.gov/ medicare-coverage-database/details/ncd-details.aspx :NCDId=211\&ncdver $=$ $2 \&$ NCAId $=92 \&$ ver $=19 \&$ NcaName $=$ Positron + Emission + Tomography $+\& b c=$ BAAAgAAAAgAA\&. Accessed December 9, 2011.

4. Centers for Medicare and Medicaid Services national coverage determinations with data collection as a condition for coverage: coverage with evidence development. Centers for Medicare and Medicaid Services Web site. Available at: https://www. cms.gov/CoverageGenInfo/03_CED.asp. Accessed December 9, 2011.

5. Hillner BE, Liu D, Coleman RE, et al. The National Oncologic PET Registry (NOPR): design and analysis plan. J Nucl Med. 2007;48:1901-1908.

6. Hillner BE, Tunuguntla R, Fratkin M, et al. Clinical decisions associated with positron emission tomography in a prospective cohort of patients with suspected or known cancer at one United States center. J Clin Oncol. 2004;22:4147-4156.

7. FDG indications: cancers and indications eligible for entry in the NOPR. National Oncologic PET Registry Web site. Available at: http://www.cancerpetregistry. org/pdf/FDG\%20Indications.pdf. Accessed December 1, 2011.

8. Gazelle GS, Kessler L, Lee DW, et al. A framework for assessing the value of diagnostic imaging in the era of comparative effectiveness research. Radiology. 2011;261:692-698.

9. Frank RA, Rucker DW, Ferguson MA, et al. Evidence requirements for innovative imaging devices: from concept to adoption. J Am Coll Radiol. 2011;8:124-131.

10. Memorandum of understanding between United States Food and Drug Administration and the Centers for Medicare and Medicaid Services. Fed Regist. 2010;75:48699-48705.

\section{Erratum}

In the article "Prognostic Significance of ${ }^{18}$ F-FDG PET Parameters and Plasma Epstein-Barr Virus DNA Load in Patients with Nasopharyngeal Carcinoma," by Chang et al. (J Nucl Med. 2012;53:21-28), the byline should have indicated that Drs. Kai-Ping Chang and Ngan-Ming Tsang contributed equally to the work. We regret the error. 